\title{
渐开线斜齿轮传动摩擦动力学耦合研究
}

\author{
邹玉静 ${ }^{1}$ 庞 峰 ${ }^{2}$ 焚智敏 ${ }^{1}$ \\ (1. 青岛科技大学机电工程学院 青岛 266061; \\ 2. 青岛市计量技术研究院 青岛 266101)
}

\begin{abstract}
摘要: 综合考虑时变啮合刚度、轴承刚度以及摩擦力等对动力学行为的影响, 基于载荷分担理论和动力学、弹流润滑理论, 建立 12 自由度斜齿轮摩擦动力学模型。采用解耦方法求解该摩擦动力学模型, 将动力学求解获得的动态轮齿作用力用于润 滑分析中, 而润滑分析获得的摩擦因数再次用于动力学分析计算中。通过实例研究了齿面摩擦学特性和动力学行为以及两者 之间的耦合关系。研究表明: 考虑耦合效应后的斜齿轮动态响应与定摩擦因数下的动态响应相比有较大不同, 且时变摩擦力 对垂直于啮合线方向的动态响应影响尤为显著; 动态载荷等对斜齿轮润滑特性影响较大, 转速接近共振转速时, 动态载荷作 用下的油膜厚度、油膜承载比例、油膜温升和摩擦因数分布规律与幅值与稳态载荷相比差异明显。动态载荷对斜齿轮润滑特 性以及时变滑动摩擦力对动态响应的影响不可忽略。
\end{abstract}

关键词: 渐开线斜齿轮; 摩擦动力学模型; 载荷分担理论; 动力学行为

中图分类号: TH132; TH117

\section{Coupling Research on Dynamical Behavior and Elastohydrodynamic Lubrication Property of Helical Gear}

\author{
ZOU Yujing $^{1}$ PANG Feng ${ }^{2}$ FAN Zhimin ${ }^{1}$ \\ (1. College of Electromechanical Engineering, Qingdao University of Science \& Technology, Qingdao 266061;
}

2. Qingdao Institute of Measurement Technology, Qingdao 266101)

\begin{abstract}
A twelve-degree-of-freedom tribo-dynamics model for helical gear pair is proposed based on load sharing theory, dynamics and elastohydrodynamic lubrication theory. Time-varying mesh stiffness, bearing stiffness, as well as the friction force are considered when establishing the model. A decoupling method of dynamics and tribology is proposed to solve the lubrication and dynamics models simultaneously. In the iterative loop, the dynamic model predicted the dynamic tooth forces to be used in the lubrication model. In return, the gear lubrication model is provided the dynamic model with periodic friction coefficient. An example gear pair is analyzed using the proposed model and method to demonstrate this coupling relationship between tribological and dynamic behaviors. The results show that the helical gear's dynamic response with consideration of coupling effect is different from the dynamic response under the constant friction factor. Friction force has significant effect on dynamic response that's perpendicular to action line. Dynamic load offects lubrication property greatly. Moreover, when the working rotational speed is approaching the resonance speed, compared with steady load, the distribution and amplitude of oil film thickness, portion of load carried by film, film temperature rise and the friction coefficient the oil film thickness is quite different. The influence of dynamic load on helical gear's lubrication property can't be ignored, so does the influence of time-varying sliding friction on dynamic response.
\end{abstract}

Key words: helical gear; tribo-dynamics model; load sharing theory; dynamic behavior

\section{0 前言}

渐开线斜齿轮因传动平稳、承载能力高等优点 广泛应用于电动汽车轮边减速器等高速场合。与传

* 山东省自然科学基金(ZR2018MEE014)和山东省重点研发计划 (2018GGX103015)资助项目。20180419 收到初稿, 20180908 收到修改稿
统汽车比, 纯电动汽车的动力源由发动机变成电机 后噪声降低, 轮边减速器的噪声会越来越明显, 而 齿轮传递误差是影响电动汽车减速器 $\mathrm{NVH}$ 性能的 重要因素 ${ }^{[1-2]}$ 。齿轮传动系统的动力学行为和润滑特 性将对整机的噪音、振动, 效率、精度和可靠性等 产生较大影响。一直以来渐开线齿轮在啮合过程中 的动力学特性、润滑特性引起了国内外学者的高度 
关注 ${ }^{[3-6]}$ 。LI 等 ${ }^{[7]}$ 建立了直齿轮的摩擦动力学疲劳 模型, 证明了摩擦动力学对齿轮疲劳损伤起重要作 用。苑士华等 ${ }^{[8]}$ 研究表明: 油膜的存在以及摩擦因 数的变化都将影响齿轮的动力学特性 ${ }^{[8]}$ 。而直齿轮 的动力学特性将影响油膜压力、油膜厚度、摩擦因 数等摩擦学特性 ${ }^{[9]}$ 。因此, 开展斜齿轮的摩擦动力 学耦合研究, 对提高斜齿轮的啮合平稳性、承载能 力和降低摩擦损耗等具有重要意义。

本文将斜齿轮传动多自由度动力学模型与混合 弹流润滑模型相结合, 建立多自由度斜齿轮摩擦动 力学模型。既考虑齿轮摩擦学特性对其动力学行为 的影响, 同时考虑动态载荷、摩擦转矩对齿面润滑 油膜的作用, 研究斜齿轮齿面的摩擦学特性和动力 学行为以及两者之间的耦合关系。

\section{1 不考虑弹流润滑效应的斜齿轮摩擦}

动力学模型

\section{1 斜齿轮多自由度动力学模型}

根据斜齿轮的啮合原理, 其系统振动具有典 型的多自由度振动特性, 除具有弯曲振动、轴向 振动和扭转振动外, 还包含轴向动态啮合力产生 的扭摆振动等。采用集中参数法建立斜齿轮传动 系统的弯-扭-轴-摆动力学分析模型, 模型具有主、 从动齿轮沿 $x 、 y 、 z$ 向平移和绕 $x 、 y 、 z$ 轴旋转的 12 个自由度, 如图 1 所示, 其中 $x$ 轴沿啮合线方 向, 以轴线方向为 $z$ 轴, 与 $x 、 z$ 两方向垂直的方 向即为 $y$ 轴。

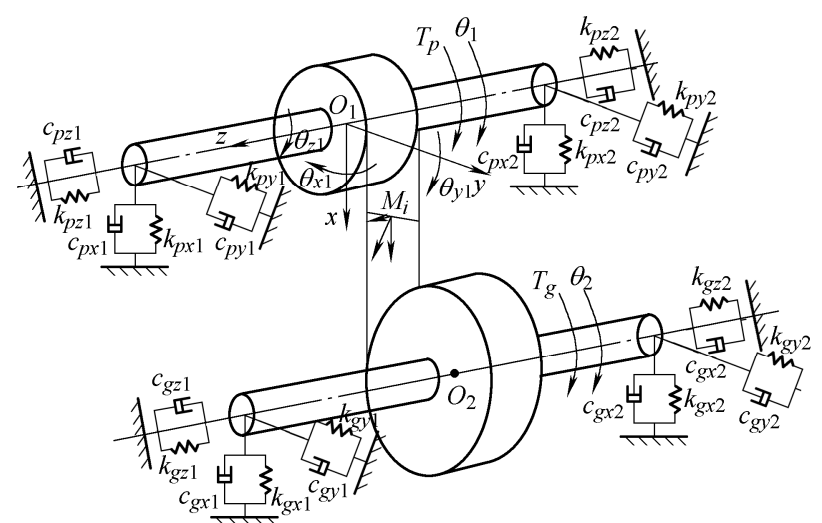

图 1 斜齿轮副 12 自由度动力学模型

其中广义位移列阵 $\boldsymbol{q}$ 可以表示为

$$
\boldsymbol{q}=\left[x_{1}, y_{1}, z_{1}, \theta_{x 1}, \theta_{y 1}, \theta_{z 1}, x_{2}, y_{2}, z_{2}, \theta_{x 2}, \theta_{y 2}, \theta_{z 2}\right]^{\mathrm{T}}
$$

建立如图 2 所示的坐标系, $z$ 轴沿着齿轮轴线 方向, $x$ 轴沿着啮合线方向。 $r_{\mathrm{b} 1}$ 和 $r_{\mathrm{b} 2}$ 分别为主从动 齿轮的基圆半径, $\beta_{\mathrm{b}}$ 为基圆螺旋角。根据图 2 可得
某啮合时刻接触线上 $M_{i}$ 点处由各位移分量产生的 法向距离为

$$
\left.\begin{array}{c}
\delta\left(M_{i}\right)=\boldsymbol{V}^{\mathrm{T}}\left(M_{i}\right) \boldsymbol{q} \\
\cos \beta_{b} \\
0 \\
\sin \beta_{b} \\
-r_{b 1} \sin \beta_{b} \\
-z \cos \beta_{b}+x \sin \beta_{b} \\
r_{b 1} \cos \beta_{b} \\
-\cos \beta_{b} \\
0 \\
-\sin \beta_{b} \\
-r_{b 2} \sin \beta_{b} \\
-x) \sin \beta_{b}+z \cos \beta_{b} \\
r_{b 2} \cos \beta_{b}
\end{array}\right)
$$

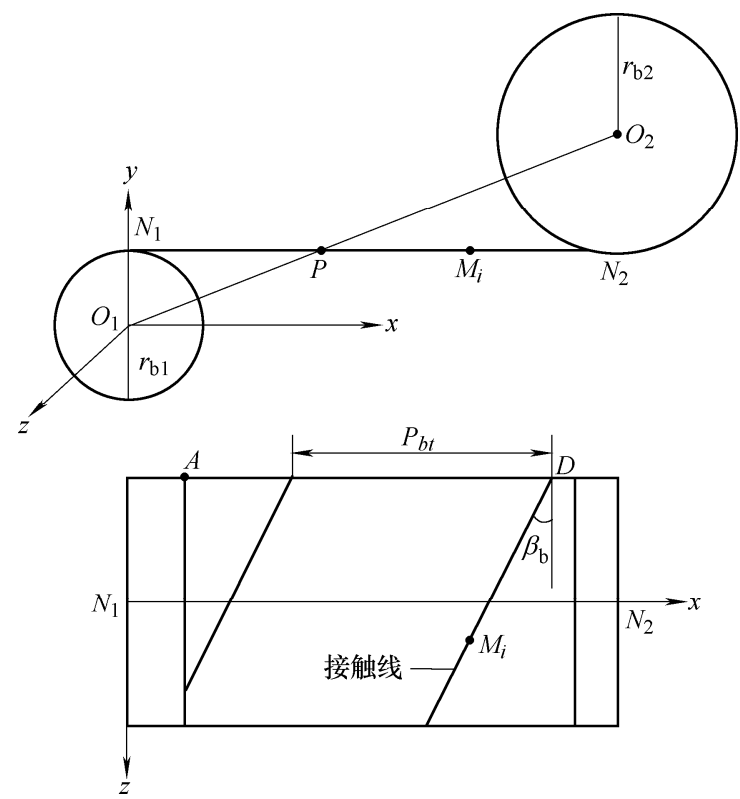

图 2 参数及坐标系定义

令 $\Delta\left(M_{i}\right)=\delta\left(M_{i}\right)-\delta_{e}\left(M_{i}\right), \delta_{e}\left(M_{i}\right)$ 为静态传递 误差, 则 $M_{i}$ 点处的法向力为

$$
\begin{gathered}
\mathrm{d} N_{i}=k_{0}(t) \Delta\left(M_{i}\right) \mathrm{d} l+c_{0}(t) \dot{\Delta}\left(M_{i}\right) \mathrm{d} l= \\
k_{0}(t)\left[\delta\left(M_{i}\right)-\delta_{e}\left(M_{i}\right)\right] \mathrm{d} l+c_{0}(t)\left[\dot{\delta}\left(M_{i}\right)-\dot{\delta}_{e}\left(M_{i}\right)\right] \mathrm{d} l
\end{gathered}
$$

式中, $k_{0}(t) 、 c_{0}(t)$ 分别为单位长度接触线上的啮合 刚度系数和阻尼系数; $\mathrm{d} l$ 为接触单元长度。

主动轮与从动轮上 $M_{i}$ 点处的啮合力以及啮合 力矩为

$$
\Delta \boldsymbol{F}_{m}\left(M_{i}\right)=-\boldsymbol{V} \mathrm{d} N_{i}
$$

$M_{i}$ 点处的切向力即摩擦力为

$$
\mathrm{d} F_{f i}=\lambda_{i} f_{i} \mathrm{~d} N_{i}
$$

式(6)方向判断系数 $\lambda_{i}=\operatorname{sgn}\left(x-x_{p}\right), f_{i}$ 为摩擦 
因数。

主动轮与从动轮上 $M_{i}$ 点处的各个方向上摩擦 力以及摩擦力矩为

$$
\begin{gathered}
\Delta \boldsymbol{F}_{f}\left(M_{i}\right)=\boldsymbol{X} \mathrm{d} F_{f i}=\lambda_{i} f_{i} \boldsymbol{X} \mathrm{d} N_{i} \\
\boldsymbol{X}\left(M_{i}\right)=\left[\begin{array}{lllllll}
0 & 1 & 0 & z & 0 & -x & 0 \\
-1 & 0 & -z & 0 & -\left(x_{g}-x\right)
\end{array}\right]^{\mathrm{T}}
\end{gathered}
$$

由式(1) (5)可得啮合时刻 $t$ 时主、从动轮各个 方向上的总啮合力和总啮合力力矩为

$$
\begin{gathered}
\boldsymbol{F}_{m}=\int_{L_{z}} \Delta \boldsymbol{F}_{m}=\boldsymbol{K}_{m} \boldsymbol{q}+\boldsymbol{C}_{m} \dot{\boldsymbol{q}}+F_{1 e} \\
\boldsymbol{C}_{m}=c_{0}(t) \int_{L_{z}}-\boldsymbol{V} \boldsymbol{V}^{T} \mathrm{~d} l \\
\boldsymbol{K}_{m}=k_{0}(t) \int_{L_{z}}-\boldsymbol{V} \boldsymbol{V}^{T} \mathrm{~d} l \\
\boldsymbol{F}_{l e}=k_{0}(t) \int_{L_{z}} \boldsymbol{V} \delta_{e}\left(M_{i}\right) \mathrm{d} l+c_{0}(t) \int_{L} \boldsymbol{V} \dot{\delta}_{e}\left(M_{i}\right) \mathrm{d} l
\end{gathered}
$$

$$
\boldsymbol{K}_{S B}=-\left(\begin{array}{cccccccccccc}
k_{1 x} & 0 & 0 & 0 & 0 & 0 & 0 & 0 & 0 & 0 & 0 & 0 \\
0 & k_{1 y} & 0 & 0 & 0 & 0 & 0 & 0 & 0 & 0 & 0 & 0 \\
0 & 0 & k_{1 z} & 0 & 0 & 0 & 0 & 0 & 0 & 0 & 0 & 0 \\
0 & 0 & 0 & k_{1 \theta x} & 0 & 0 & 0 & 0 & 0 & 0 & 0 & 0 \\
0 & 0 & 0 & 0 & k_{1 \theta y} & 0 & 0 & 0 & 0 & 0 & 0 & 0 \\
0 & 0 & 0 & 0 & 0 & 0 & 0 & 0 & 0 & 0 & 0 & 0 \\
0 & 0 & 0 & 0 & 0 & 0 & k_{2 x} & 0 & 0 & 0 & 0 & 0 \\
0 & 0 & 0 & 0 & 0 & 0 & 0 & k_{2 y} & 0 & 0 & 0 & 0 \\
0 & 0 & 0 & 0 & 0 & 0 & 0 & 0 & k_{2 z} & 0 & 0 & 0 \\
0 & 0 & 0 & 0 & 0 & 0 & 0 & 0 & 0 & k_{2 \theta x} & 0 & 0 \\
0 & 0 & 0 & 0 & 0 & 0 & 0 & 0 & 0 & 0 & k_{2 \theta y} & 0 \\
0 & 0 & 0 & 0 & 0 & 0 & 0 & 0 & 0 & 0 & 0 & 0
\end{array}\right)
$$

式中, $k_{i j}$ 为轴-轴承综合刚度, $i=1,2$, 分别代表主 动轮和从动轮; $j=x, y, z, \theta_{x}, \theta_{y}$, 分别代表各个 方向。

主、从动轮上各个方向的阻尼力和力矩为

$$
\boldsymbol{F}_{C}=\boldsymbol{C}_{S B} \dot{\boldsymbol{q}}
$$

$\boldsymbol{C}_{S B}=-\operatorname{diag}\left(c_{1 x}, c_{1 y}, c_{1 z}, c_{1 \theta_{x}}, c_{1 \theta_{y}}, 0, c_{2 x}, c_{2 y}, c_{2 z}, c_{2 \theta_{x}}, c_{2 \theta_{y}}, 0\right)$

根据牛顿第二定律可得

$$
[M] \ddot{\boldsymbol{q}}=\boldsymbol{F}_{0}+\boldsymbol{F}_{m}+\boldsymbol{F}_{f}+\boldsymbol{F}_{S B}+\boldsymbol{F}_{C}
$$

$$
\boldsymbol{F}_{0}=\left[\begin{array}{llllllllllll}
0 & 0 & 0 & 0 & 0 & T_{p} & 0 & 0 & 0 & 0 & 0 & T_{g}
\end{array}\right]^{\mathrm{T}}(13
$$

式(12)可进一步写为

$$
[M] \ddot{\boldsymbol{q}}=[C] \dot{\boldsymbol{q}}+[K] \boldsymbol{q}+\boldsymbol{F}_{0}+\boldsymbol{F}_{1 e}+\boldsymbol{F}_{2 e}
$$

$[M]=\operatorname{diag}\left(m_{1}, m_{1}, m_{1}, I_{1}, I_{1}, I_{p 1}, m_{2}, m_{2}, m_{2}, I_{2}, I_{2}, I_{p 2}\right)$

$$
[K]=\boldsymbol{K}_{m}+\boldsymbol{K}_{f}+\boldsymbol{K}_{S B}
$$

$L_{z}$ 为 $t$ 时刻时斜齿轮总的接触线长度, 计算方 法见参考文献[6]。由式(1) (7)可得主、从动轮各个 方向上的总摩擦力和总摩擦力力矩为

$$
\begin{gathered}
\boldsymbol{F}_{f}=\int_{L_{z}} \Delta \boldsymbol{F}_{f}=\boldsymbol{K}_{f} \boldsymbol{q}+\boldsymbol{C}_{f} \dot{\boldsymbol{q}}+\boldsymbol{F}_{2 e} \\
\boldsymbol{K}_{f}=k_{0}(t) \int_{L_{z}} \lambda_{i} f_{i} \boldsymbol{X} \boldsymbol{V}^{T} \mathrm{~d} l \\
\boldsymbol{C}_{f}=c_{0}(t) \int_{L_{z}} \lambda_{i} f_{i} \boldsymbol{X} \boldsymbol{V}^{T} \mathrm{~d} l \\
\boldsymbol{F}_{2 e}=-k_{0}(t) \int_{L_{z}} \lambda_{i} f_{i} \boldsymbol{X} \delta_{e}\left(M_{i}\right) \mathrm{d} l- \\
c_{0}(t) \int_{L_{z}} \lambda_{i} f_{i} \boldsymbol{X} \dot{\delta}_{e}\left(M_{i}\right) \mathrm{d} l
\end{gathered}
$$

轴/轴承刚度产生的恢复力对主动轮和从动轮 产生的作用力和力矩为

$$
\boldsymbol{F}_{S B}=\boldsymbol{K}_{S B} \boldsymbol{q}
$$

轴/轴承刚度矩阵为

$$
[C]=\boldsymbol{C}_{m}+\boldsymbol{C}_{f}+\boldsymbol{C}_{S B}
$$

\section{2 渐开线斜齿轮啮合刚度计算}

齿轮的啮合刚度取决于轮齿的弯曲变形、剪切 变形、接触变形和轮体的轴向变形等。对于斜齿轮 传动, 齿面接触线长度按照 “点一线一点” 的方式 变化, 因此不会产生刚度突变, 但仍会引起刚度激 励, 轮齿啮合刚度具有明显的时变性和周期性。CAI 给出单对齿啮合刚度的近似表达式, 得到了广泛采 用。根据 CAI 的研究, 单对齿的啮合刚度为 ${ }^{[10]}$

$$
\begin{gathered}
k_{s}(t)=k_{p} \exp \left(C_{\beta}|X|^{3}\right) \\
X=\frac{t-\varepsilon_{\gamma} t_{c} / 2}{\left(\varepsilon_{\alpha} t_{c} / 2 m_{n}\right) \times H}
\end{gathered}
$$

式中, $X$ 为啮合位置; $k_{p}$ 为节圆位置处刚度; $C_{\beta}$ 为 螺旋角系数; $H$ 为齿全高。

$k_{p} 、 C_{\beta}$ 与节圆位置处螺旋角 $\beta_{0}$ 的关系分别为 
$C_{\beta}=0.322 \times\left(\beta_{0}-5\right)+(0.23 B / H-23.26)$

$k_{p}=\left[(-0.166 B / H+0.08)\left(\beta_{0}-5\right)+44.5\right] \times(B / H) \times m_{n}$
CAI 将式(17)进一步改进, 使其适用于变位修 形齿轮, 具体表达式为

$$
k_{p}=\frac{B}{c_{p}+c_{1}\left(\frac{1}{z_{v 1}}+\frac{1}{z_{v 2}}\right)+c_{2}\left(\frac{y_{1}}{z_{v 1}}+\frac{y_{2}}{z_{v 2}}\right)+c_{3}\left(\frac{1}{z_{v 1}^{2}}+\frac{1}{z_{v 2}^{2}}\right)+c_{4}\left(y_{1}+y_{2}\right)+c_{5}\left(y_{1}^{2}+y_{2}^{2}\right)}
$$

$$
c_{p}=\frac{H / m_{n}}{(-0.166 B / H+0.08)\left(\beta_{0}-5\right)+44.5}
$$

$$
c_{1}=-0.00854 \quad c_{2}=-0.11654 \quad c_{3}=2.9784
$$

$$
c_{4}=-0.00635 \quad c_{5}=0.00529
$$

式中, $B$ 为齿宽; $m_{\mathrm{n}}$ 为模数; $z_{v 1}, z_{v 2}$ 为主、从动 斜齿轮的当量齿数; $y_{1}, y_{2}$ 为两斜齿轮的变位系数。

\section{2 渐开线斜齿轮混合弹流润滑模型}

基于载荷分担、弹流润滑和粗粘线接触理论, 建 立计及表面粗䊅度的斜齿轮传动混合弹流润滑模型 ${ }^{[6]}$ 。

\section{1 渐开线斜齿轮啮合模型}

斜齿轮的齿面接触线和轴线不平行, 存在一个 倾斜角 $\beta_{\mathrm{b}}$ 。如图 3 所示, 渐开线斜齿轮的啮合面与 两齿轮的基圆柱相切, 其齿面接触线与切平面内的 轮齿迹线成一夹角 $\Delta$, 其值为 ${ }^{[11]}$

$$
\cos \Delta=\frac{\cos \beta^{\prime}}{\cos \beta_{b}}
$$

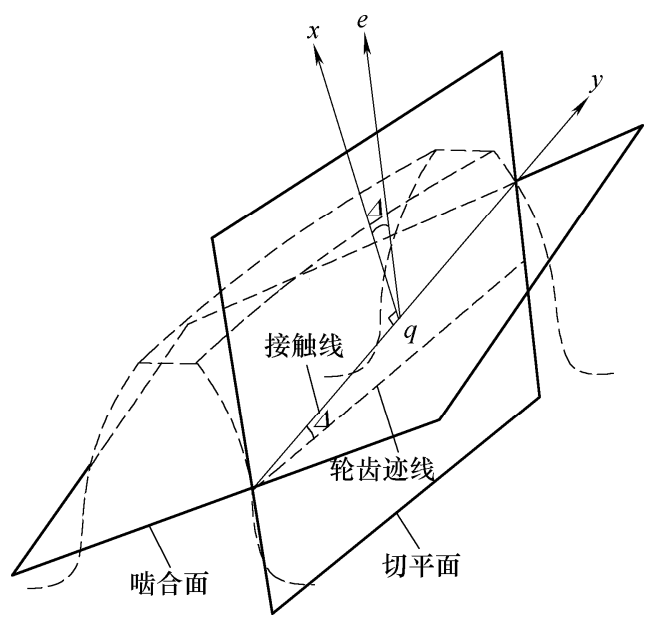

图 3 斜齿轮几何图形

将斜齿轮沿接触线分成许多薄片, 每一薄片看 成是具有当量角速度 $\hat{\omega}=\omega \cos \Delta$ 的直齿圆柱齿轮 ${ }^{[2]}$ 。 根据欧拉方程即可得到任一接触点处的曲率半径和 表面速度 ${ }^{[11]}$ 。

\section{2 混合弹流润滑模型}

\subsection{1 载荷分担理论}

绝大多数齿轮传动在混合弹流状态下工作, 根 据 JOHNSON 等的研究 ${ }^{[13]}$, 齿面法向载荷 $F_{d}$ 由润滑 油膜和粗糙峰共同承担, 齿面摩擦力 $F_{f}$ 则由润滑油
膜摩擦力 $F_{f h}$ 和粗粘峰接触摩擦力 $F_{f a}$ 共同组成, 即

$$
\begin{gathered}
\left\{\begin{array}{c}
F_{d}=F_{h}+F_{a} \\
F_{f}=F_{f h}+F_{f a}
\end{array}\right. \\
F_{h}=\frac{F_{d}}{\gamma_{1}} \quad F_{a}=\frac{F_{d}}{\gamma_{2}}
\end{gathered}
$$

式中, $1 / \gamma_{1}$ 为润滑油膜承载比例; $1 / \gamma_{2}$ 为粗粘峰承 载比例。

\subsection{2 粗楉峰接触压力方程}

同一润滑状态下, Gelinek-Schipper 接触理论 ${ }^{[14]}$ 与 Greenwood-Tripp 接触理论 ${ }^{[15]}$ 计算的粗糙峰压力 值相等 ${ }^{[16]}$

$$
\begin{gathered}
\frac{1}{\gamma_{2}} \sqrt{\frac{F_{d} E^{\prime}}{2 \pi B R}}\left[1+\left(1.558\left(n_{s} \gamma_{2} R \sqrt{\beta_{s} R}\right)^{0.0337} \times\right.\right. \\
\left.\left.\left(\frac{\sigma_{s}}{R}\right)^{-0.442} W^{0.4757}\right)\right]^{-0.5882}= \\
\frac{8 \sqrt{2}}{15} \pi n_{s}^{2} \beta_{s}^{1.5} \sigma_{s}^{2.5} E^{\prime} F_{5 / 2}\left(\frac{h_{c t}-d_{d}}{\sigma_{s}}\right)
\end{gathered}
$$

具体参数定义可见文献[16]。

\subsection{3 油膜厚度方程}

AKBARZADEH 等根据 MOE 方程 ${ }^{[17]}$ 得到考虑 表面粗粘度的直齿轮等温量纲一中心油膜厚度公式 为 $^{[16]}$

$$
\begin{gathered}
H_{C}=\left[\gamma_{1}^{s / 2}\left(H_{R I}^{7 / 3}+\left(\gamma_{1}\right)^{-14 / 15} H_{E I}^{7 / 3}\right)^{3 s / 7}+\right. \\
\left.\gamma_{1}^{-s / 2}\left(H_{R P}^{-7 / 2}+H_{E P}^{-7 / 2}\right)^{-2 s / 7}\right]^{1 / s}\left(\gamma_{1}\right)^{1 / 2} \\
s=\frac{1}{5}\left[7+8 \exp \left(\frac{-2\left(\gamma_{1}\right)^{-2 / 5} H_{E I}}{H_{R I}}\right)\right]
\end{gathered}
$$

MOE 方程的膜厚对应于牛顿流体，JANG 基于 Carreau 流变模型对线接触弹流中心膜厚修正公式 进行修正 ${ }^{[18]}$

$$
\phi_{c}=\frac{h_{c}}{h_{c}^{N}}=\left(1+0.75(1+s r) \Gamma^{1 /\left(1+0.22 s_{r}\right)}\right)^{3.1(1-n)^{1.6}}
$$

引入热修正系数 $C_{t}$ 考虑齿轮啮入端润滑油温度 变化对油膜厚度的影响 ${ }^{[19]}$

$$
C_{t}=\frac{h_{c t}}{h_{c}}=\frac{1}{1+0.0766 G^{0.687} W^{0.447} L_{c}^{0.527} e^{0.875 s_{r}}}
$$




$$
L c=\frac{\mu_{0} \beta_{f} u_{r}^{2}}{k_{f}}
$$

式中, $h_{c t}$ 为非牛顿流体热油膜厚度; $k_{f}$ 为润滑剂 的热传导系数; $\beta_{f}$ 为温黏系数。其余参数见参考文 献[16]。

根据式(20) (25)可求得不同啮合位置时的 $\gamma_{1}$ 、 $\gamma_{2} 、 h_{c t}{ }^{[6]}$ 。

\subsection{4 润滑油流变模型本构方程及黏压温方程}

基于幂函数非牛顿流变模型的理论分析更能真 实地预测润滑油膜在弹流工况下的润滑特性 ${ }^{[20-21]}$, 因此本文采用常用的幂函数型 Carreau-Yasuda 非牛 顿流变模型, 其本构方程为 ${ }^{[22-23]}$

$$
\mu_{e}=\tau / \dot{\gamma}=\mu\left[1+\left(\frac{\mu \dot{\gamma}}{G_{c r}}\right)^{2}\right]^{(n-1) / 2}
$$

式中, $\mu$ 为低压剪切黏度; $\dot{\gamma}$ 为切应变率。

选用 Doolittle-Tait 自由体积黏度模型来描述弹 流工况下润滑膜的黏压关系, 其黏压温方程 ${ }^{[22]}$ 为

$$
\mu=\mu_{r} \exp \left[B_{0} R_{0}\left(\frac{1+\varepsilon\left(T-T_{r}\right)}{\frac{V}{V_{r}}-R_{0}\left(1+\varepsilon\left(T-T_{r}\right)\right)}-\frac{1}{1-R_{0}}\right)\right]
$$

采用 TAIT 状态方程 ${ }^{[24]}$ 描述润滑油体积随着压 力的变化关系

$$
\frac{V}{V_{R}}=\left[1-\frac{1}{1+K_{0}^{\prime}} \ln \left(1+\frac{p}{K_{0}}\left(1+K_{0}^{\prime}\right)\right)\right]\left(1+\alpha_{V}\left(T-T_{R}\right)\right)
$$

\subsection{5 能量方程及边界条件}

根据能量方程及界面边界条件来确定润滑油膜 的温度场。

考虑粗粘峰接触的线接触弹流润滑能量方 程 ${ }^{[25]}$ 为

$$
k_{f} \frac{\partial^{2} T}{\partial z^{2}}=-\mu_{e}\left(\frac{\partial u}{\partial z}\right)^{2}+c_{f} \rho_{f} u \frac{\partial T}{\partial x}-\frac{f_{a} p_{a} u_{s}}{h_{c t}}
$$

将式(26)对油膜厚度求导, 可得速度方程

$$
\begin{gathered}
\mu_{e} \frac{\partial^{2} u}{\partial z^{2}}+\mu_{e}^{\prime} \frac{\partial u}{\partial z}=\tau^{\prime} \\
\mu_{e}^{\prime}=\frac{\partial \mu_{e}}{\partial z} \quad \tau^{\prime}=\frac{\partial \tau}{\partial z}
\end{gathered}
$$
程计算 ${ }^{[26]}$

油膜与齿轮轮齿接触面的温度可根据 Jaeger 方

$$
\left\{\begin{array}{l}
T(x, 0)=T_{0}+\left.\frac{k_{f}}{\sqrt{\pi \rho_{1} c_{1} k_{1} u_{1}}} \int_{-b}^{x} \frac{\partial T}{\partial x}\right|_{z=0} \frac{\mathrm{d} s}{\sqrt{x-s}} \\
T(x, h)=T_{0}+\left.\frac{k_{f}}{\sqrt{\pi \rho_{2} c_{2} k_{2} u_{2}}} \int_{-b}^{x} \frac{\partial T}{\partial x}\right|_{z=h} \frac{\mathrm{d} s}{\sqrt{x-s}}
\end{array}\right.
$$

通过求解能量方程、速度方程和界面温度方程 ${ }^{[27]}$, 即可得到最终的温度场和剪应力分布。

\subsection{6 摩擦因数求解}

总摩擦因数为

$$
f=\frac{F_{f}}{F_{d}}=\frac{F_{f h}+F_{f a}}{F_{d}}=\frac{B \int_{x_{i n}}^{x_{\text {out }}} \tau \mathrm{d} x}{F_{d}}+\frac{f_{a}}{\gamma_{2}}
$$

根据第 2.2.2 节 2.2.5 节求解得到粗䊁峰承载 比例 $1 / \gamma_{2}$ 和剪应力 $\tau$, 将其代入式(32), 即可得到 啮合点处的总摩擦因数 $f$ 。

\section{3 斜齿轮摩擦-动力学耦合}

如前所述, 在对齿轮传动系统进行分析时, 必须考虑摩擦学特性对其动力学行为的影响, 以 及动态啮合力和摩擦转矩对齿面润滑油膜的作用 和温升效应, 即需要进行齿轮弹流润滑特性和动 力学行为耦合研究。因此综合考虑斜齿轮轮齿刚 度的时变特性、表面粗䊁度及摩擦转矩的作用, 将斜齿轮 12 自由度动力学模型与斜齿轮的热混合 弹流润滑模型相结合, 建立斜齿轮的多自由度摩 擦动力学模型。

首先求解动力学方程, 获得不同啮合时刻的齿 面动载荷、综合曲率半径、卷吸速度、相对滑动速 度等动力学参数; 然后进行斜齿轮混合弹流润滑分 析, 得到新的摩擦因数。将新的摩擦因数重新代入 动力学方程, 将迭代后新得到的动态载荷与上次动 力学分析计算后求解得到的动态载荷进行对比, 反 复迭代计算直至动载荷收敛, 终止计算。耦合求解 计算流程简图如图 4 所示。

\section{4 结果及讨论}

根据建立的摩擦动力学模型, 与非耦合情况进 行对比研究耦合系统的动力学特性和润滑特性。

\section{1 考虑动载荷的斜齿轮润滑特性分析}

以表 1 中齿轮副为研究对象 ${ }^{[28]}$ (润滑油特性 参数见表 $2^{[29]}$ ), 采用 Carreau 非牛顿流体, 耦合动 态载荷条件下油膜厚度、油膜承载比例、油膜温 升和摩擦因数变化对比分别见图 5 7, 并与准稳 态载荷情况 (见参考文献 [6]) 进行对比。转速为 $8000 \mathrm{r} / \mathrm{min}$ 时, 准稳态载荷和耦合动态载荷条件 下的油膜厚度、摩擦因数和油膜温升变化对比分 别见图 $8 \sim 10$ 。 


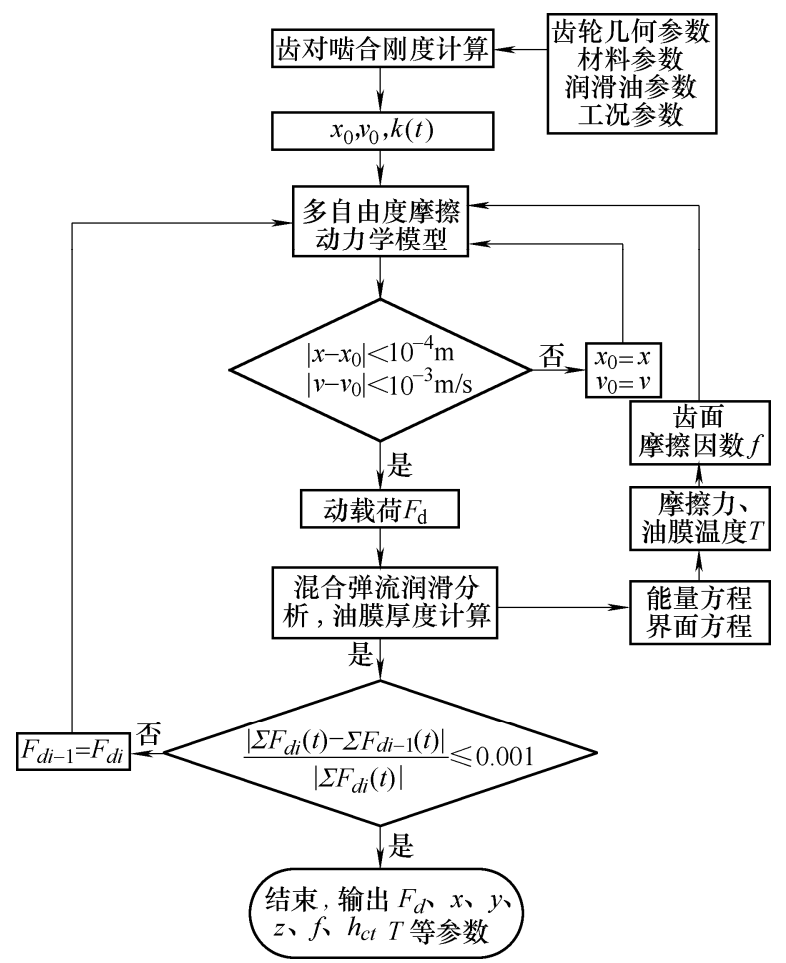

图 4 求解流程图

表 1 齿轮传动参数和性能

\begin{tabular}{|c|c|c|}
\hline 参数/性能 & 主动轮 & 从动轮 \\
\hline 齿数 $z$ & 25 & 31 \\
\hline 齿顶圆直径 $d_{a} / \mathrm{mm}$ & 85.85 & 104.9 \\
\hline 齿根圆直径 $d_{f} / \mathrm{mm}$ & 71.4 & 90.45 \\
\hline 转速 $n /(\mathrm{r} / \mathrm{min})$ & 3000 & \\
\hline 转矩 $T /(\mathrm{N} \cdot \mathrm{m})$ & 360 & \\
\hline 端面模数 $m_{t} / \mathrm{mm}$ & \multicolumn{2}{|c|}{3.175} \\
\hline 端面压力角 $\alpha_{t}{ }^{\circ} \mathrm{C}$ & \multicolumn{2}{|c|}{25} \\
\hline 螺旋角 $\beta /{ }^{\circ} \mathrm{C}$ & \multicolumn{2}{|c|}{21.5} \\
\hline 齿宽 $B / \mathrm{mm}$ & \multicolumn{2}{|c|}{31.75} \\
\hline 中心距 $a / \mathrm{mm}$ & \multicolumn{2}{|c|}{88.9} \\
\hline 弹性模量 $E / \mathrm{GPa}$ & \multicolumn{2}{|c|}{210} \\
\hline 泊松比 $v$ & \multicolumn{2}{|c|}{0.3} \\
\hline 粗粘度均方根值 $\sigma_{s} / \mu \mathrm{m}$ & \multicolumn{2}{|c|}{0.4} \\
\hline 粗粘峰半径 $\beta_{s} / \mu \mathrm{m}$ & \multicolumn{2}{|c|}{6} \\
\hline 密度 $\rho /\left(\mathrm{kg} / \mathrm{m}^{3}\right)$ & \multicolumn{2}{|c|}{7800} \\
\hline 热传导系数 $k /(\mathrm{W} /(\mathrm{m} \cdot \mathrm{K}))$ & \multicolumn{2}{|c|}{47} \\
\hline 比热容 $c /(\mathrm{J} / \mathrm{kg} \cdot \mathrm{K})$ & \multicolumn{2}{|c|}{460} \\
\hline 粗䄳峰摩擦因数 $f \mathrm{a}$ & \multicolumn{2}{|c|}{0.1} \\
\hline
\end{tabular}

表 2 润滑油特性参数

\begin{tabular}{lc}
\hline \multicolumn{1}{c}{ 性能 } & 数值 \\
\hline 热传导系数 $k_{f} /(\mathrm{W} /(\mathrm{m} \cdot \mathrm{K}))$ & 0.14 \\
密度 $\rho_{f} /\left(\mathrm{kg} / \mathrm{m}^{3}\right)$ & 864 \\
比热容 $c_{f} /(\mathrm{J} / \mathrm{kg} \cdot \mathrm{K})$ & 2000 \\
$\mu_{\mathrm{R}} / \mathrm{Pa} . \mathrm{s}$ & 1.42 \\
$T_{R}{ }^{\circ} \mathrm{C}$ & 75 \\
$B_{0}$ & 4.423 \\
$R_{0}$ & 0.6694 \\
$\varepsilon /{ }^{\circ} \mathrm{C}^{-1}$ & -0.0008 \\
$K_{0}{ }^{2}$ & 12.82 \\
$K_{00} / \mathrm{GPa}$ & 11.5 \\
$\beta_{k} / \mathrm{K}^{-1}$ & 0.0006 \\
$\alpha_{v} /{ }^{\circ} \mathrm{C}^{-1}$ & 0.0007 \\
$n$ & 0.74 \\
$G_{c r} / \mathrm{KPa}^{-1}$ & 31 \\
$\alpha / \mathrm{GPa}^{-1}$ & 19 \\
\hline
\end{tabular}

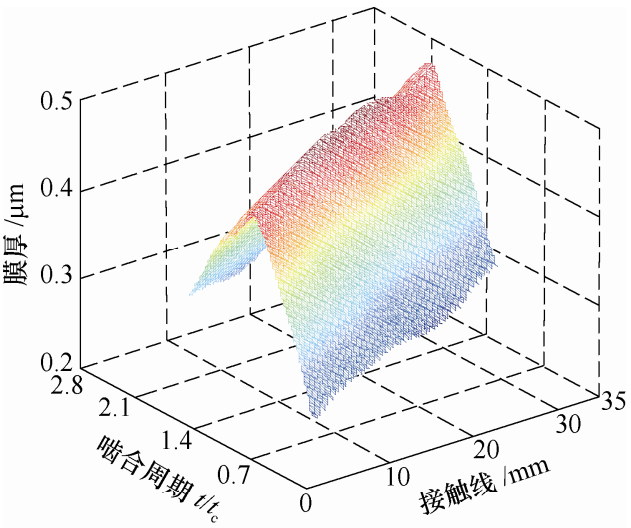

(a) 膜厚

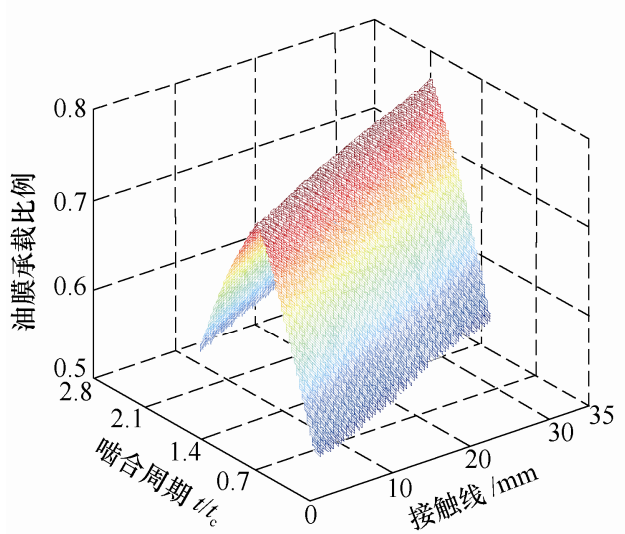

(b) 油膜承载比例
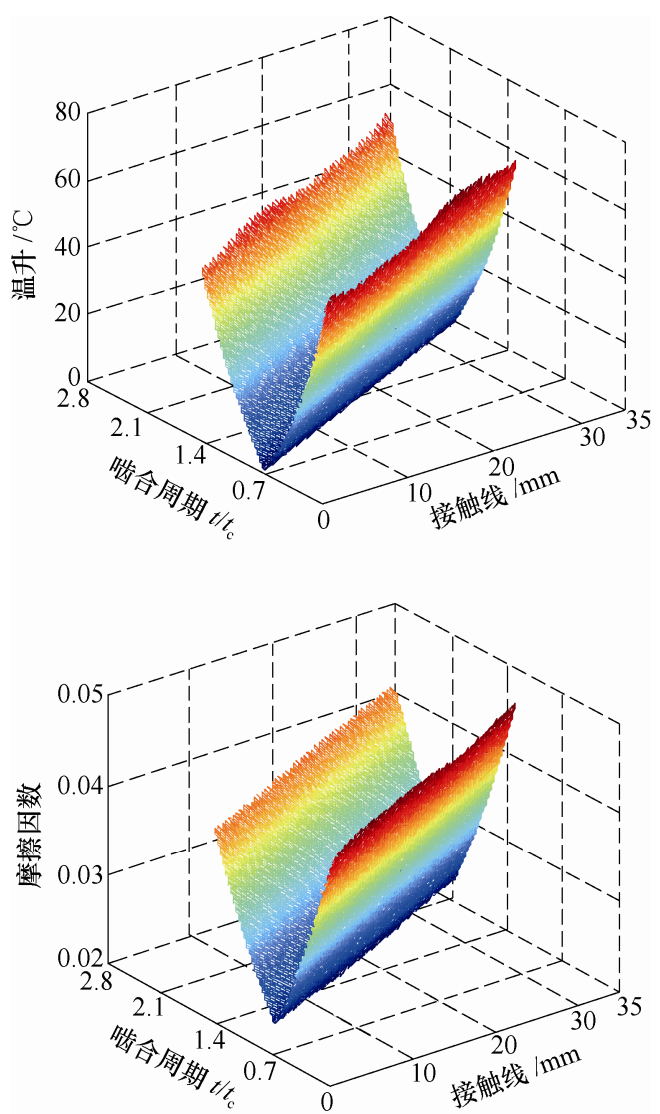

图 5 耦合动载荷下斜齿轮 Carreau 流体润滑特性结果 (3000 r/min) 


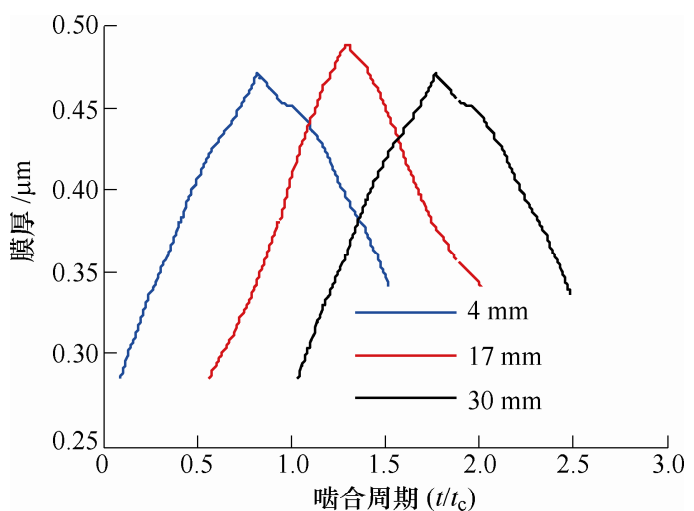

(a) $3000 \mathrm{r} / \mathrm{min}$



(b) $8000 \mathrm{r} / \mathrm{min}$

图 6 耦合动载荷下不同接触线位置的油膜厚度随时间变化

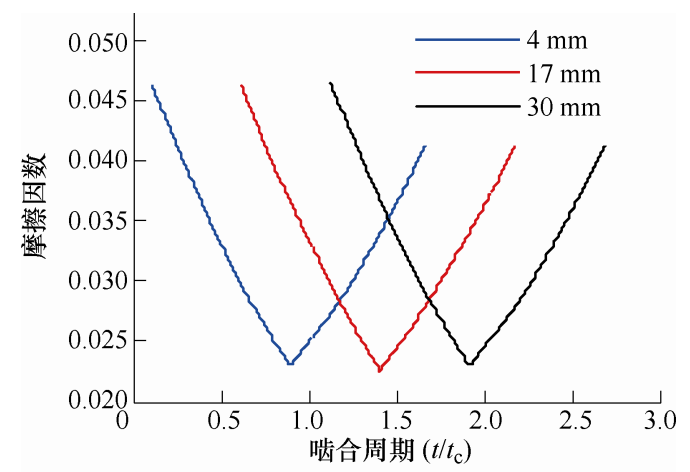

(a) $3000 \mathrm{r} / \mathrm{min}$

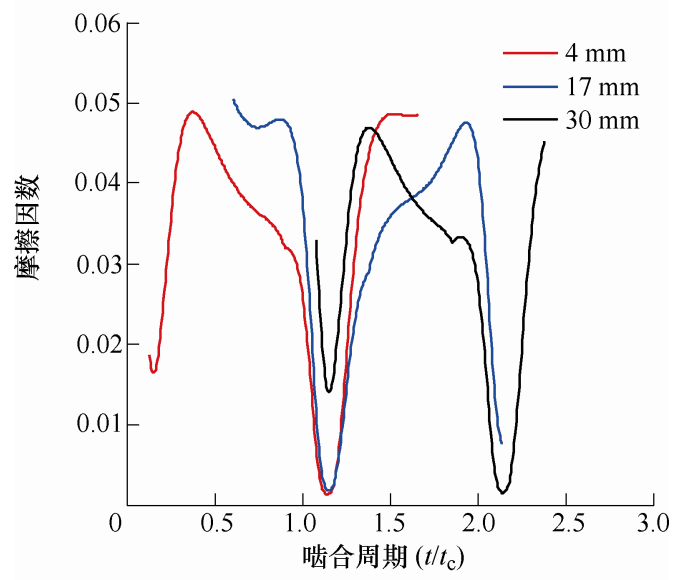

(b) $8000 \mathrm{r} / \mathrm{min}$

图 7 耦合动载荷下不同接触线位置的 摩擦因数随时间变化

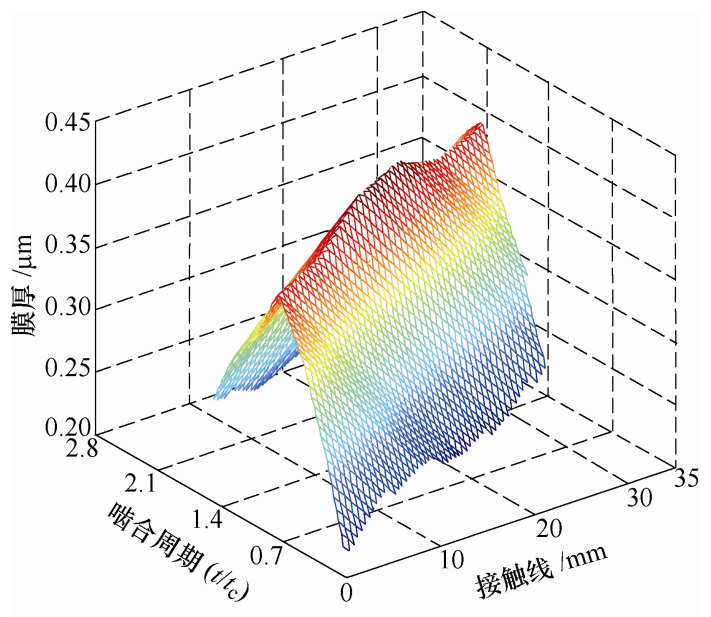

(a) 准稳态载荷

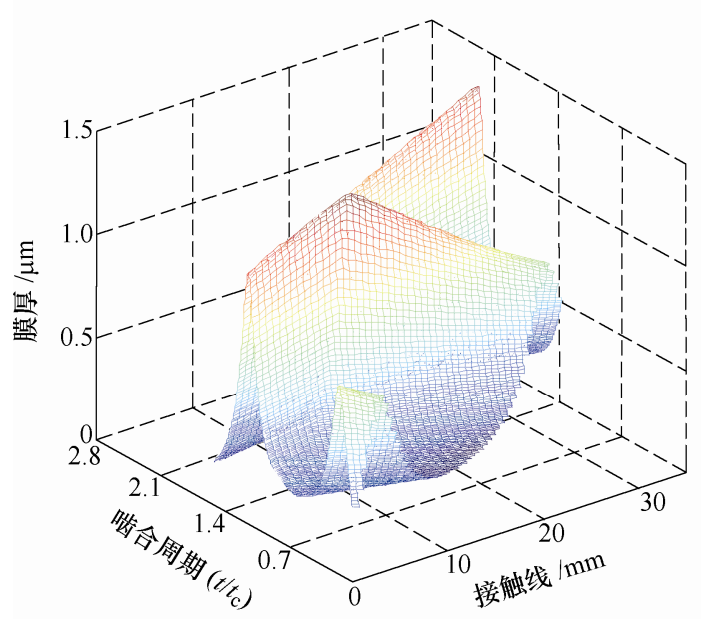

(b) 耦合载荷

图 8 两种载荷下的油膜厚度结果

根据图 5 10 并结合文献[6]对比可看出, 所研 究斜齿轮对在给定工况下(转矩 $360 \mathrm{~N} \cdot \mathrm{m}$, 转速 $3000 \mathrm{r} / \mathrm{min}$ ), 耦合载荷作用时的斜齿轮副油膜厚 度、油膜承载比例、油膜温升和摩擦因数分布规律与 稳态载荷作用下相似, 且幅值相差不大, 与稳态载荷 作用时相比, 油膜厚度略有变薄, 油膜承载比例稍微 降低, 油膜温升略有升高, 因此摩擦因数略有增大。

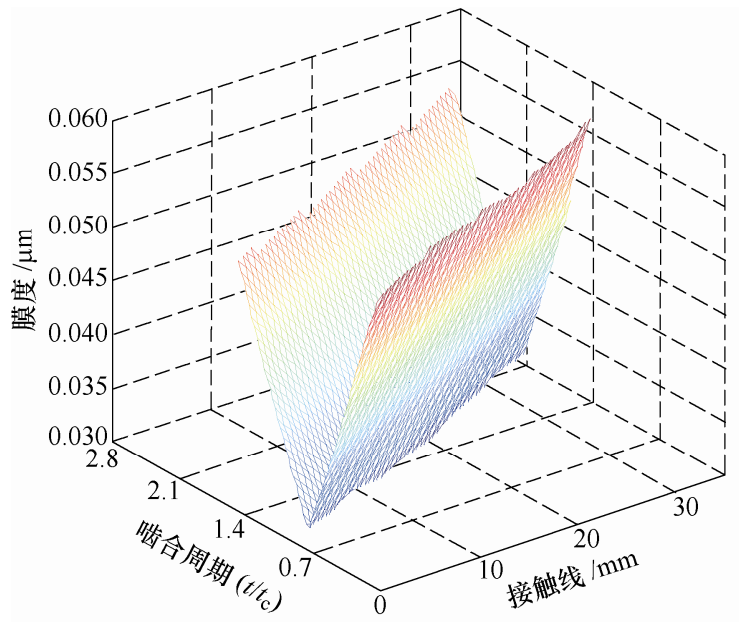

(a) 准稳态载荷 


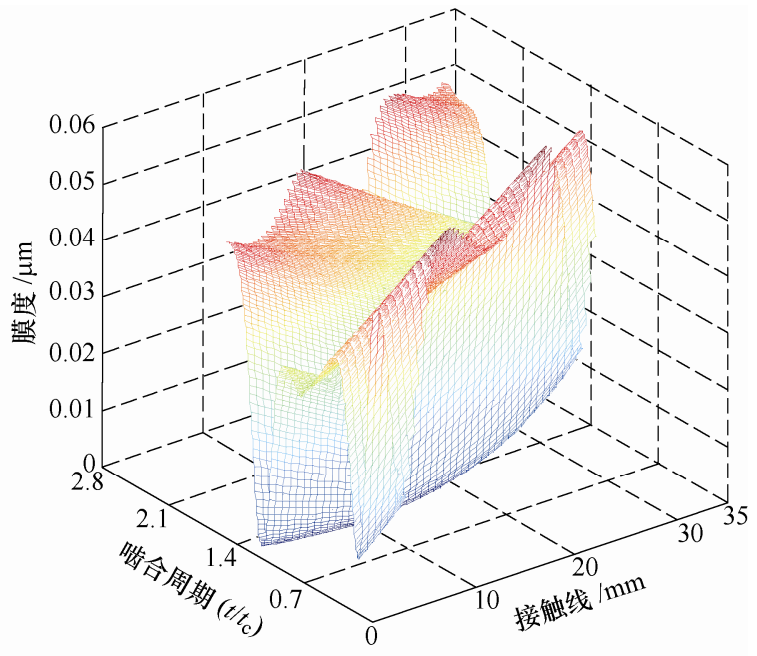

（b）耦合载荷

图 9 两种载荷下的摩擦因数结果

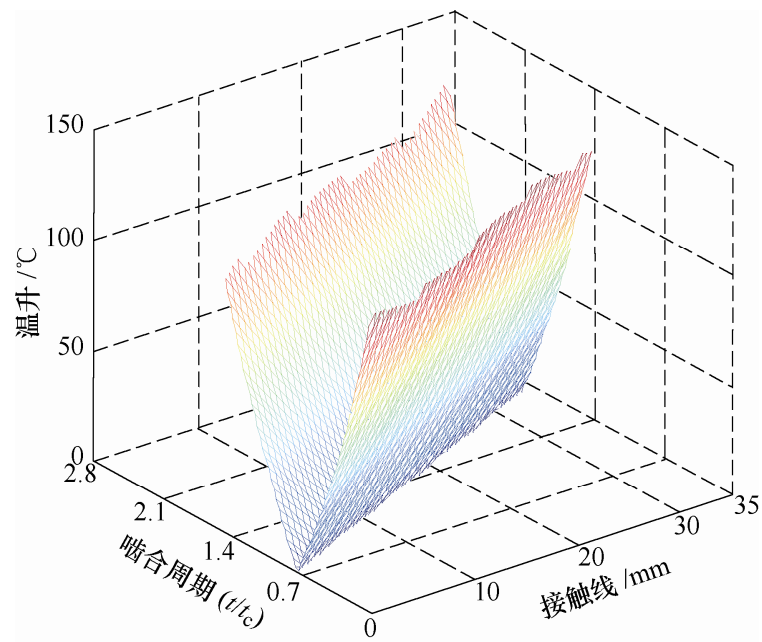

(a) 准稳态载荷

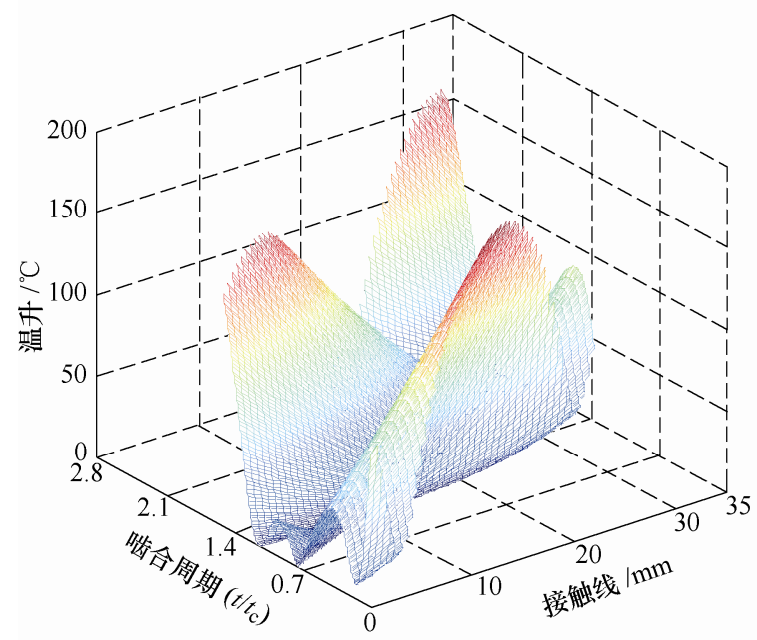

(b) 耦合载荷

图 10 两种载荷下的油膜温升结果

当转速增加至 $8000 \mathrm{r} / \mathrm{min}$, 两种载荷作用下的 油膜厚度等分布差异比较显著, 这是因为润滑特性 是动态载荷、综合曲率半径和相对滑动速度等共同
作用的结果, 而转速是影响齿轮系统齿面动载荷等 动态特性的重要因素之一。低速时，齿面动载荷均 值与稳态分布接近。随着转速的增加, 齿面动载荷 与稳态载荷分布的差异愈加显著。当转速为 8000 $\mathrm{r} / \mathrm{min}$ 时, 系统接近共振转速, 动载荷幅值在节点位 置附近达到最大值, 且动态载荷随时间波动较大, 使得油膜厚度、油膜温升、摩擦因数等随时间波动 也较大。

这说明齿轮转速等工况以及动态载荷对斜齿轮 润滑特性有较大的影响。为准确反映齿轮传动系统 的润滑性能，应该将齿轮的弹流润滑特性和齿轮的 动力学行为耦合起来研究。

\section{2 考虑弹流效应的斜齿轮动力学特性分析}

图 11 14 为采用耦合模型的时变摩擦因数和 定摩擦因数(摩擦因数 $f$ 取定值 0.035 ) 时所研究斜齿 轮对在给定工况下(转矩 $360 \mathrm{~N} \cdot \mathrm{m}$, 转速 $3000 \mathrm{r} / \mathrm{min}$ ) 主动斜齿轮 $x 、 y 、 z$ 方向位移及动态啮合力响应图。


图 11 主动斜齿轮 $x$ 向位移随时间的变化曲线

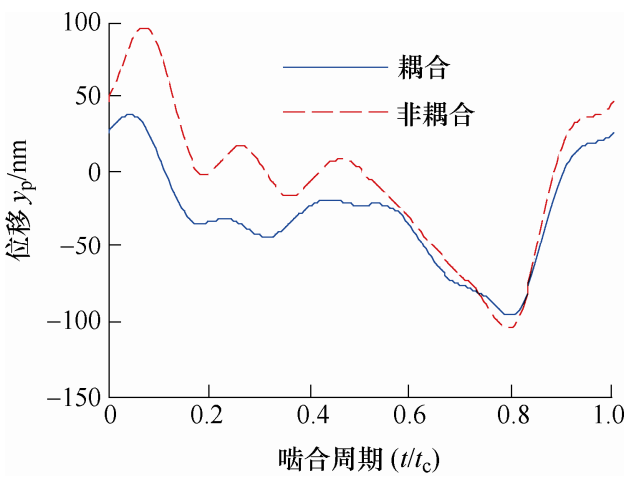






图 12 主动轮 $y$ 向位移随时间的变化曲线
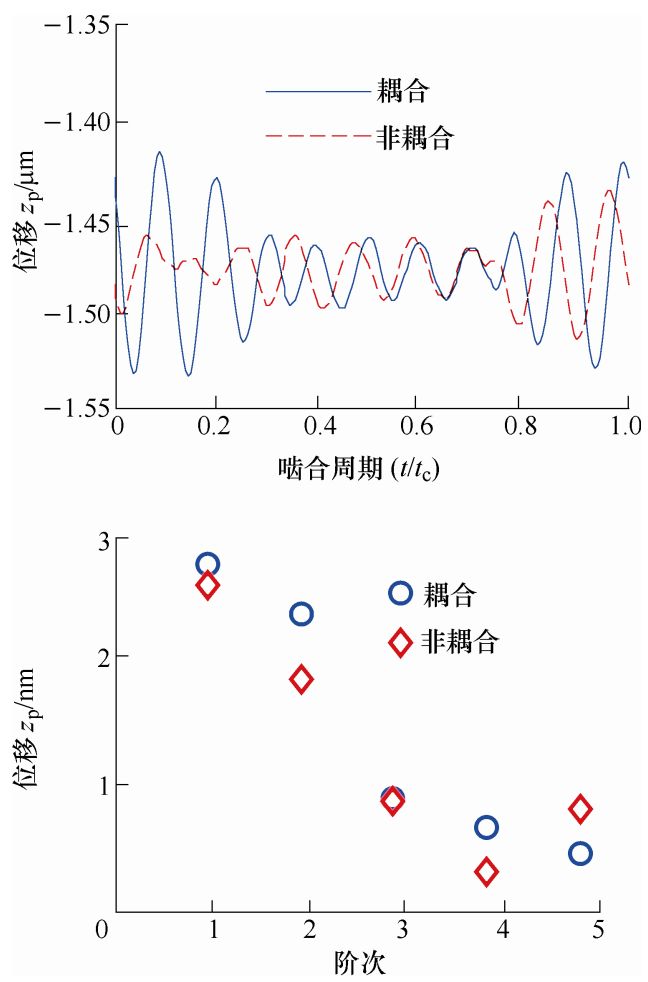

图 13 主动轮 $z$ 向位移随时间的变化曲线

从图 11 14 可看出, 给定工况下耦合模型和非 耦合模型的主动轮各向位移和动态啮合力波形和幅 值相差较大。

在垂直啮合线方向, 时变摩擦因数使主动轮的 振幅减小, 尤其是第 1 阶谐波。这表明垂直啮合线 方向的动态响应受摩擦效应影响最为显著。

主动轮的轴向位移比较高, 且振幅大小取决 于摩擦因数大小, 时变摩擦力增加了第 2 阶谐波 的幅值。

综上所述, 时变摩擦力对斜齿轮动力学响应的 影响不能忽略, 摩擦力对垂直于啮合线方向的位移 影响显著, 对啮合线方向和轴线方向的位移有轻微 影响。

\section{5 结论}

(1) 动态特性是齿轮啮合刚度、传动误差、摩
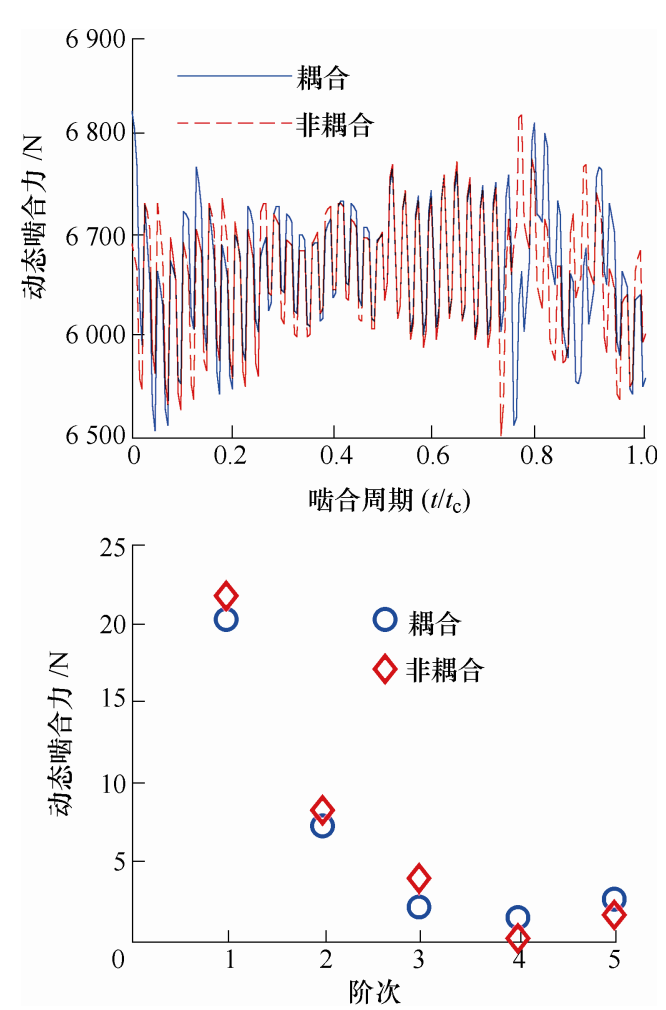

图 14 动态啮合力随时间的变化曲线

擦因数等因素综合作用的结果。摩擦力对斜齿轮传 动垂直于啮合线方向的位移影响显著。耦合模型下 的动态响应与定摩擦因数下的动态响应相比有较 大不同, 时变摩擦力对斜齿轮动力学行为的影响 较显著。

（2）润滑特性受动载荷、综合曲率半径和相对 滑动速度等因素共同影响。转速低时, 耦合模型下 斜齿轮的油膜厚度、油膜承载比例、油膜温升和摩 擦因数分布规律和幅值同稳态载荷作用相差不大, 转速接近共振转速时则差异显著。表明转速、动态 载荷等对斜齿轮润滑特性影响较大。

\section{参 考 文 献}

[1] 丁金刚, 纪弘祥. 电动汽车减速器齿轮修形对传递误差 影响的研究 [J]. 车辆与动力技术, 2017(4): 33-36.

DING Jingang, JI Hongxiang. Influence of gear modification on transmission error of $\mathrm{EV}$ reducer[J]. Vehicle \& Power Technology, 2017(4): 33-36.

[2] 潘晓东, 刘祥环, 黎超. 纯电动汽车高速齿轮传动 $\mathrm{NVH}$ 性能优化研究[J]. 重庆理工大学学报, 2017(11): 25-31.

PAN Xiaodong, LIU Xianghuan, LI Chun. Investigation on NVH performance optimization of BEV high speed gear transmission[J]. Journal of Chongqing University of Technology, 2017(11): 25-31.

[3] BEILICKE R, BOBACH L, BARTEL D. Transient thermal elastohydrodynamic simulation of a DLC Coated helical gear pair considering limiting shear stress behavior 
of the lubricant[J]. Tribology International, 2016, 97 : 136-150.

[4] LEE J, YOON M, BOO K, et al. Prediction of transmission error using dynamic analysis of a helical gear[J]. Transactions of the Korean Society of Mechanical Engineers - A, 2016, 40(12): 1005-1011.

[5] SAXENA A, PAREY A, CHOUKSEY M. Time varying mesh stiffness calculation of spur gear pair considering sliding friction and spalling defects[J]. Engineering Failure Analysis, 2016，70: 200-211.

[6] 邹玉静, 常德功. 基于载荷分担理论的渐开线斜齿轮热 混合弹流润滑分析 [J]. 摩擦学学报, 2015, 5(4): 495-504.

ZOU Yujing , CHANG Degong. Mixed thermoelastohydrodynamic analysis of helical gears based on load sharing concept [J]. Tribology, 2015, 5(4): 495-504.

[7] LI S, ANISETTI A. A tribo-dynamic contact fatigue model for spur gear pairs[J]. International Journal of Fatigue, 2017, 98: 81-91.

[8] 苑士华, 董辉立, 胡纪滨, 等. 考虑油膜润滑作用的渐 开线齿轮动载荷分析 [J]. 机械工程学报, 2012, 48(19): 10-16.

YUAN Shihua, DONG Huili, HU Jibin, et al. Dynamic loading analysis of involute gears considering lubrication performance[J]. Journal of Mechanical Engineering, 2012, 48(19): 10-16.

[9] 邹玉静, 常德功. 渐开线直齿轮的动力学与弹流润滑耦 合[J]. 航空动力学报, 2016, 31(8): 2010-2020.

ZOU Yujing, CHANG Dechang. Study on coupling dynamics and elastohydrodynamic lubrication of spur gear[J]. Journal of Aerospace Power, 2016, 31(8): 2010-2020.

[10] CAI Y. Simulation on the rotational vibration of helical gears in consideration of the tooth separation phenomenon (a new stiffness function of helical involute tooth pair[J]. Journal of Mechanical Design, 1995, 117(3): 460-469.

[11] LITVIN F L. Gear geometry and applied theory[M]. New Jersey: Prentice Hall, Englewood Cliffs, 1994.

[12] XU H. Development of a generalized mechanical efficiency prediction methodology for gear pairs[D]. Athens: Ohio State University, 2005.

[13] JOHNSON K L, GREENWOOD J A, POON S Y. A simple theory of asperity contact in elastohydrodynamic lubrication [J]. Wear, 1972, 19(1): 91-108.

[14] GELINCK E R M, SCHIPPER D J. Deformation of rough line contacts[J]. Journal of tribology, 1999, 121(3): 449-454.

[15] GREENWOOD J A, TRIPP J H. The contact of two nominally flat rough surfaces $[\mathrm{J}]$. Proceedings of the Institution of Mechanical Engineers, 1970, 185(1): 625-633.

[16] AKBARZADEH S, KHONSARI M M. Thermoelastohydrodynamic analysis of spur gears with consideration of surface roughness[J]. Tribology Letters, 2008, 32(2): 129-141.

[17] MOES H. Optimum similarity analysis with applications to elastohydrodynamic lubrication [J]. Wear, 1992, 159(1): 57-66.

[18] JANG J Y, KHONSARIL M M. Correction factor formula to predict the central and minimum film thickness for shear-thinning fluids in EHL [J]. Journal of Tribology, 2008, 130(024501): 1-4 .

[19] HSU C H, LEE R T. An efficient algorithm for thermal elastohydrodynamic lubrication under rolling/sliding line contacts[J]. Journal of tribology, 1994, 116(4): 762-769.

[20] BAIR S, QURESHI F. The generalized newtonian fluid model and elastohydrodynamic film thickness[J]. Journal of Tribology, 2003, 125: 70-75

[21] BAIR S, VERGNE P, QUERRY M. A unified shear-thinning treatment of both film thickness and traction in EHD[J]. Tribology Letters, 2005, 18(2): $145-152$

[22] BAIR S S. High pressure rheology for quantitative elastohydrodynamics[M]. London: Elsevier, 2007.

[23] YASUDA K Y, ARMSTRONG R C, COHEN R E. Shear flow properties of concentrated solutions of linear and star branched polystyrenes[J]. Rheologica Acta, 1981, 20(2): 163-178.

[24] DOOLITTLE A K. Studies in Newtonian Flow. II. The dependence of the viscosity of liquids on free-space[J]. Journal of Applied Physics, 2004, 22(12): 1471-1475.

[25] MASJEDI M, KHONSARI M M. Theoretical and experimental investigation of traction coefficient in line-contact EHL of rough surfaces[J]. Tribology International, 2014, 70: 179-189.

[26] JAEGER J C. Moving surfaces of heat and the temperature at sliding contacts[J]. Proc. R. Soc. N.S.W., 1942, 56: 203-224.

[27] 李朵朵. 齿轮传动动态弹流润滑的数值计算模型研究 [D]. 太原: 太原理工大学, 2004.

LI Duoduo. A calculating model study for the 
elatohydrodynamic lubrication of the transmission of the gear in mesh [D]. Taiyuan: Taiyuan University of Technology, 2004

[28] HE S, GUNDA R, SINGH R. Inclusion of sliding friction in contact dynamics model for helical gears[J]. Journal of Mechanical Design, 2007, 129: 1064-1075.

[29] ANURADHA P, KUMAR P. Effect of lubricant selection on EHL performance of involute spur gears[J]. Tribology
International, 2012, 50: 82-90.

作者简介: 邹玉静, 女, 1977 年出生, 博士, 主要研究方向为齿轮传动 与润滑。

E-mail: zouyujing-007@163.com

庞峰, 男, 1977 年出生, 硕士。主要研究方向为计量测试技术。

E-mail: 1snada@163.com

焚智敏(通信作者), 男, 1963 年出生, 博士, 教授。主要研究方向为齿 轮系统动力学。

E-mail: zmfan@163.com 